\title{
A Case of Reactive Arthritis in a Healthy Adult
}

\author{
Joseph Raco, MD' - Norhan Shamloul, MD² - Matthew Lipinski, DO³
}

A 32-year-old man presented to the emergency department with a several-day history of low back pain, right knee pain, and left foot pain. He had been treated several weeks prior to presentation for a sexually transmitted infection (STI) but could not recall which organism or antibiotic was involved.

He had no significant medical history. He had no recent abdominal pain, diarrhea, bloating, headache, myalgias, or neck stiffness.

\section{Physical examination}

His vital signs were within normal limits. He had several pinpoint erythematous patches and papules on his forearms and hyperkeratotic psoriasiform plaques on the plantar aspects of his feet; there was also desquamation of his first toe with dactylitis, which was significantly tender to palpation (Figure 1). His right knee had a large effusion and limited range of motion because of severe pain.

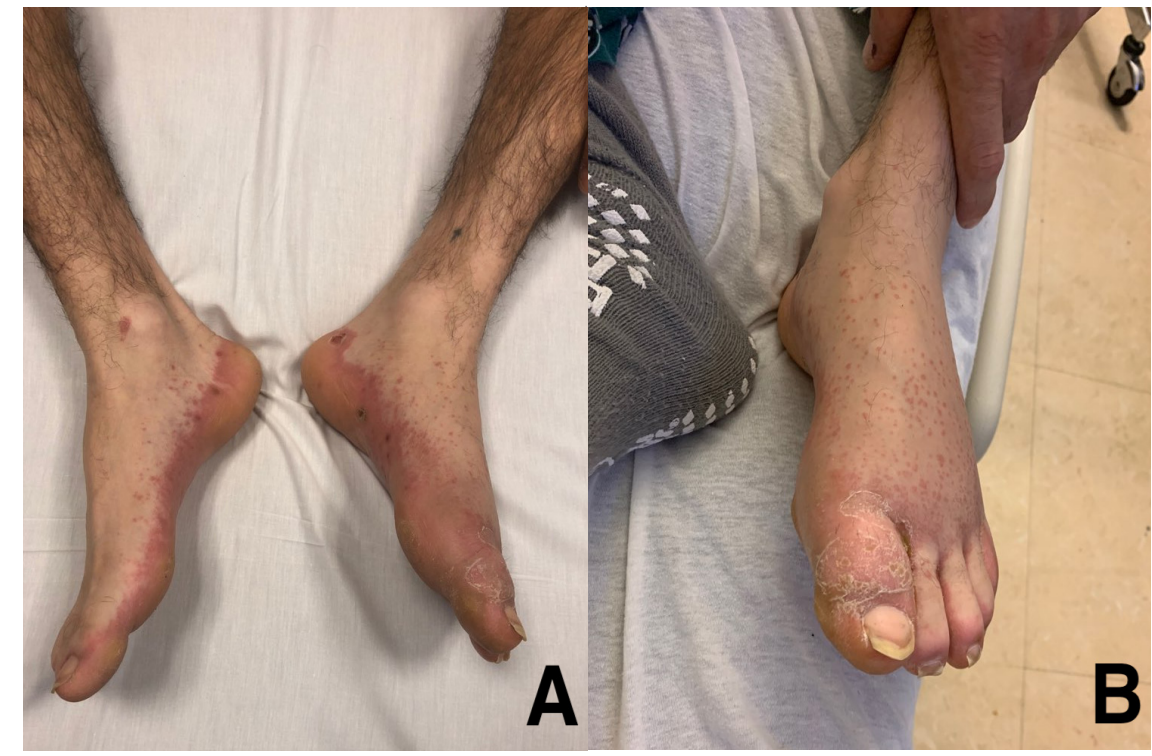

Figure 1. Hyperkeratotic psoriasiform plaques were noted on the plantar aspects of the patient's feet $(A)$. There was also desquamation of his first toe with dactylitis (B).

\section{Diagnostic testing}

Laboratory evaluation revealed no leukocytosis, an elevated platelet count of $972 \times 10^{3} / \mu \mathrm{L}$, and an elevated erythrocyte

\section{AFFILIATIONS:}

'Department of Internal Medicine, Penn State Health Milton S. Hershey Medical Center, Hershey, Pennsylvania

${ }^{2}$ Department of Dermatology, Penn State Health Milton S. Hershey Medical Center, Hershey, Pennsylvania

${ }^{3}$ Department of Internal Medicine, Mercy Hospital Oklahoma City, Oklahoma City, Oklahoma

\section{CITATION:}

Raco J, Shamloul N, Lipinski M. A case of reactive arthritis in a healthy adult. Consultant. Published online February 2, 2022. doi:10.25270/con.2022.02.00003

Received August 17, 2021. Accepted September 1, 2021.

\section{DISCLOSURES:}

The authors report no relevant financial relationships.

\section{CORRESPONDENCE:}

Matthew Lipinski, DO, Mercy Hospital Oklahoma City, 4300 West Memorial Road, Oklahoma City, OK 73120 (mlipinskido@gmail.com) sedimentation rate of $55 \mathrm{~mm} / \mathrm{hr}$. Arthrocentesis of the left knee was performed, results of which revealed 25,000 white blood cells $/ \mathrm{mm}^{3}$, no crystals, and no bacteria. Serum test results were negative for rheumatoid factor, anti-cyclic citrullinated peptide antibody, HIV, rapid plasma reagin, fluorescent treponemal antibodies, Rickettsia, Borrelia, Anaplasma, and Ehrlichia. A urine test returned negative results for Chlamydia trachomatis, Ureaplasma urealyticum, and Neisseria gonorrhoeae. A stool test returned negative results for all pathogenic organisms. Radiographs of the lumbar spine, right knee, and left foot demonstrated intraarticular effusions in the foot and knee.

\section{Patient outcome}

The patient was admitted to the hospital with uncontrolled pain and recurrent right knee effusion. His treatment includ- 


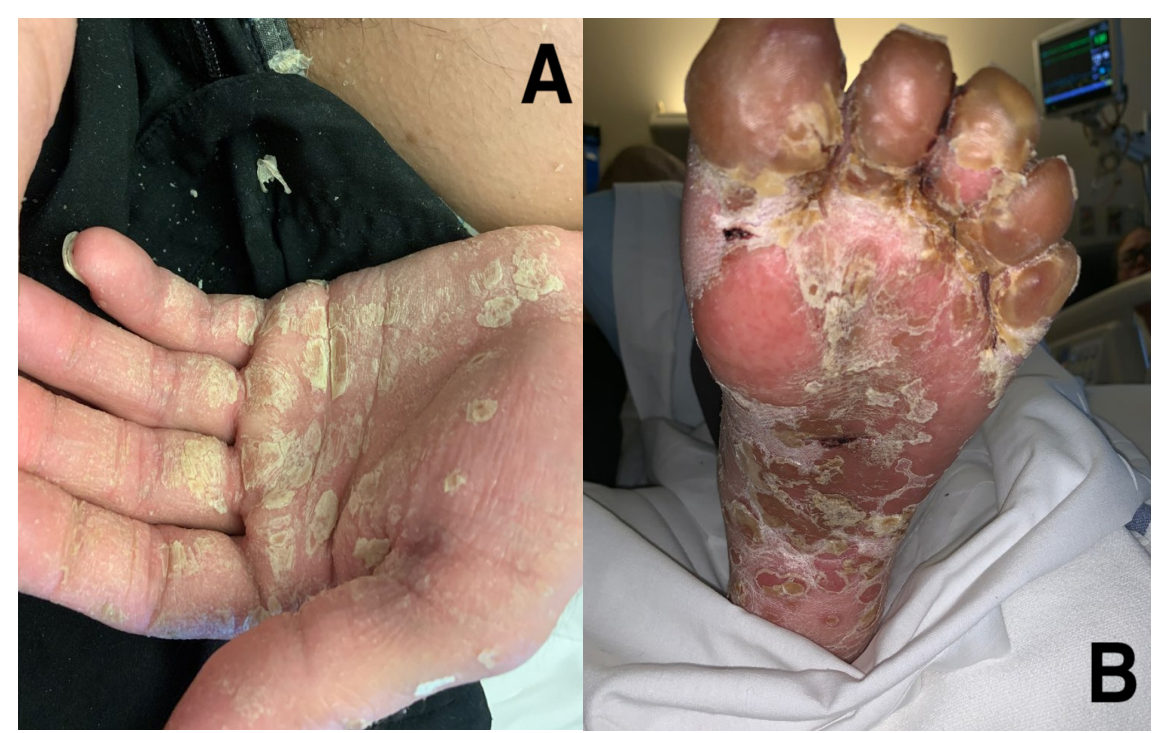

Figure 2. Pustular and scaling hyperkeratotic plaques had developed, followed by desquamation of his palms (A) and soles (B).

ed nonsteroidal anti-inflammatory drugs (NSAIDs) and intraarticular prednisone. On hospital day 11 , he developed pustular and scaling hyperkeratotic plaques followed by desquamation of his palms and soles (Figure 2). Given the classic findings of keratoderma blenorrhagicum and seronegative arthritis, as well as the patient's recently treated STI, reactive arthritis was diagnosed. Because of his severe pain, the patient required a several-week course of a systemic glucocorticoid taper to resolve his symptoms. He was discharged from the hospital after 2 weeks.

\section{Discussion}

Reactive arthritis was initially described in 1916 by Hans Reiter as Reiter syndrome..$^{12}$ It is classically described as a form of seronegative spondyloarthropathy that typically follows a gastrointestinal or urogenital infection. It is an inflammatory condition with the potential to present as a multiorgan systemic disease. The incidence of reactive arthritis differs depending on the preceding triggering infection. It affects only $1.4 \%$ of children following a Clostridioides difficile infection but affects about $4 \%$ to $8 \%$ of patients after a genital chlamyd- ia infection. ${ }^{3,4}$ Results of a systematic review showed that reactive arthritis had occurred in 12 cases per 1000 Salmonella infections, 9 cases per 1000 Campylobacter infections, and 12 cases per 1000 Shigella infections. ${ }^{3}$ The relative risk for developing reactive arthritis is highest among women and adults aged 20 to 40 years. ${ }^{3,5}$ Some studies have found that White individuals are more frequently affected, possibly because of the higher frequency of human leukocyte antigen B27 in this population, as the antigen is prevalent in $50 \%$ to $80 \%$ of patients with reactive arthritis., ${ }^{3,5}$

The pathogenesis of reactive arthritis remains incompletely understood. Multiple hypotheses have been proposed to describe the pathogenesis of the disorder. Two of the most prominent theories include the arthritogenic pathogen hypothesis, which proposes an aberrant antigen presentation leads to autoimmunity and inflammation, and the heavy chain homodimer hypothesis, which postulates that heavy chains activate natural killer cells, B cells, and T cells that in turn cause a more generalized inflammatory response. $^{3}$

Clinical presentation of reactive arthritis is heterogeneous. Some patients may be asymptomatic while others exhibit multisystem involvement. The onset of the disorder is frequently preceded by the symptoms of the inciting infection. The symptoms are dependent on the infectious organism but frequently include diarrhea, gastrointestinal distress, cervicitis, urethritis, or dysuria, which typically occur 1 to 3 weeks prior to the clinical symptoms of reactive arthritis. ${ }^{5}$ Patients may present with asymmetric oligoarthritis affecting the large and small joints of the lower extremities, enthesitis, bursitis, or dactylitis. Extraarticular manifestations are particularly prominent in the setting of genitourinary infection and include conjunctivitis, episcleritis, erythema nodosum, and keratoderma blennorhagicum. ${ }^{3,5,6}$ The classic triad of reactive arthritis is conjunctivitis, urethritis, and arthritis. Keratoderma blennorrhagicum presents as hyperkeratotic, psoriasiform plaques often on acral surfaces such as the soles of the feet and, less commonly, the palms of the hands. ${ }^{3,7}$

Diagnosis of reactive arthritis requires a high index of suspicion, as it can often be missed or misdiagnosed. The history and physical examination findings for the disorder are central to the diagnosis. The American College of Rheumatology had proposed diagnostic criteria for reactive arthritis in 1999, but they have not been validated or widely adopted. ${ }^{5,8}$ However, the criteria do represent the clinical features of reactive arthritis well. Proposed major criteria include the findings of a monoarthritis or oligoarthritis, as well as a preceding symptomatic infection consistent with enteritis or urethritis. ${ }^{5}$ Minor criteria include evidence of a triggering infection such as positive urine or urethral/cervical swab for $C$ trachomatis, positive stool cultures for enteric pathogens, or evidence of persistent synovial infection. ${ }^{3,5,8}$ Although keratoderma blennorrhagicum is not included in the criteria, it can be a valuable clue to the diagnosis particularly when combined with the proper clinical context. The differential diagnosis of reactive arthritis is broad and includes disseminated gonococcal 
infection, septic arthritis, osteoarthritis, Lyme disease, Behçet disease, psoriatic arthritis, and poststreptococcal arthritis. ${ }^{3}$

There is no universally recommended treatment for reactive arthritis. Treatment is dependent on the suspected source of each patient's underlying infection. The efficacy of antibiotics for management of reactive arthritis was assessed in a 2013 systematic review, results of which showed no significant benefit of antibiotics but did show significantly increased rates of gastrointestinal adverse reactions. ${ }^{9}$ However, if the patient is believed to have acute $C$ trachomatis genitourinary infection-related reactive arthritis, treatment of the underlying infection with a macrolide or tetracycline is recommended..$^{10}$ For management of acute arthritisrelated pain, NSAIDs are generally recommended as first-line therapy, but this regimen needs further study. ${ }^{11}$ For arthritis pain that is refractory to NSAIDs, intraarticular or systemic glucocorticoid therapy is frequently prescribed, but evidence supporting usage is limited.12 Recently, the use of biologic agents, specifically anti-tumor necrosis factor a agents, has been studied for management of reactive arthritis. A 2020 review concluded that this therapy requires further study before an official recommendation could be made. $^{13}$

Reactive arthritis is thought to be a self-limiting disease, although up to $63 \%$ of patients with reactive arthritis develop chronic arthritis. ${ }^{13}$ As such, health care providers need to be clear with their patients regarding the natural history of the disease and treatment expectations. Generally, the same therapies used for management of acute reactive arthritis are used for chronic arthropathies and are primarily based on the use of NSAIDs, although the use of sulfasalazine and biologic therapies are under further study. ${ }^{14}$

\section{Conclusions}

Reactive arthritis is a rare disease that requires clinicians to maintain a high index of suspicion. Careful history taking and thorough physical examination are the basis of diagnosis. While it is not a diagnosis of exclusion, it is prudent to perform serologic and radiographic testing to evaluate for more-easily treatable causes of patients' symptoms. As was the case with our patient, many patients who develop reactive arthritis experience preceding infectious symptoms a few weeks prior to presentation of monoarthritis, oligoarthritis, or polyarthritis. Treatment guidelines are not well defined, and management is largely supportive with the use of systemic NSAIDs, antibiotics, and intraarticular glucocorticoids. However, none of these treatments have strong evidence supporting their use. As such, management is tailored on a case-bycase basis.

\section{References}

1. Amor B. Reiter's syndrome. Diagnosis and clinical features. Rheum Dis Clin North Am. 1998;24(4):677-vii. https://doi.org/10.1016/ s0889-857x(05)70037-5

2. Ngaruiya CM, Martin IB. A case of reactive arthritis: a great masquerader. Am J Emerg Med. 2013;31(1). https://doi.org/10.1016/j. ajem.2012.04.019

3. Schmitt SK. Reactive arthritis. Infect Dis Clin North Am. 2017;31(2):265-277. https:// doi.org/10.1016/j.idc.2017.01.002

4. Horton DB, Strom BL, Putt ME, Rose CD, Sherry DD, Sammons JS. Epidemiology of Clostridium difficile infection-associated reactive arthritis in children: an underdiagnosed, potentially morbid condition. JAMA Pediatr. 2016;170(7):e160217. https://doi. org/10.1001/jamapediatrics.2016.0217

5. Selmi C, Gershwin ME. Diagnosis and classification of reactive arthritis. Autoimmun Rev. 2014;13(4-5):546-549. https://doi. org/10.1016/j.autrev.2014.01.005

6. Kiss S, Letko E, Qamruddin S, Baltatzis S, Foster CS. Long-term progression, prognosis, and treatment of patients with recurrent ocular manifestations of Reiter's syndrome. Ophthalmology. 2003;110(9):17641769. https://doi.org/10.1016/s01616420(03)00620-1

7. Florell SR, Krueger GG, Egan CA. Keratoderma blennorrhagicum. $N$ Engl J Med. 2003;349(24):2367-2368. https://doi. org/10.1056/nejm200312113492425

8. Sieper J, Braun J, Kingsley GH. Report on the fourth international workshop on reactive arthritis. Arthritis Rheum. 2000;43(4):720-734. https://doi.org/10.100 2/1529-0131(200004)43:4\%3C720::aid-anr2\%3E3.0.co;2-8

9. Barber CE, Kim J, Inman RD, Esdaile JM, James MT. Antibiotics for treatment of reactive arthritis: a systematic review and metaanalysis. J Rheumatol. 2013;40(6):916928. https://doi.org/10.3899/jrheum.121192

10. Bardin T, Enel C, Cornelis F, et al. Antibiotic treatment of venereal disease and Reiter's syndrome in a Greenland population. Arthritis Rheum. 1992;35(2):190-194. https://doi. org/10.1002/art.1780350210

11. Juvakoski T, Lassus A. A double-blind cross-over evaluation of ketoprofen and indomethacin in Reiter's disease. Scand J Rheumatol. 1982;11(2):106-108. https://doi. org/10.3109/03009748209098171

12. Morris $\mathrm{D}$, Inman RD. Reactive arthritis: developments and challenges in diagnosis and treatment. Curr Rheumatol Rep. 2012;14(5):390-394. https://doi.org/10.1007/ s11926-012-0280-4

13. Zeng $\mathrm{H}$, Luo $B$, Zhang $Y$, Xie Z, Ye Z. Treatment of reactive arthritis with biological agents: a review. Biosci Rep. 2020;40(2):BSR20191927. https://doi. org/10.1042/bsr20191927

16. Hannu T. Reactive arthritis. Best Pract Res Clin Rheumatol, 2011;25(3):347-357. https:// doi.org/10.1016/j.berh.2011.01.018 